\title{
Brassinolide interacts with auxin and ethylene in the root gravitropic response of maize (Zea mays)
}

\author{
Soo Chul Chang ${ }^{\mathrm{a}, 1}$, Young-Soo Kim ${ }^{\mathrm{b}, 1}$, Jin Young Lee ${ }^{\mathrm{c}}$, Peter B. Kaufman ${ }^{\mathrm{d}}$, Ara Kirakosyan ${ }^{\mathrm{d}}$, Hye Sup Yun ${ }^{\mathrm{b}}$,

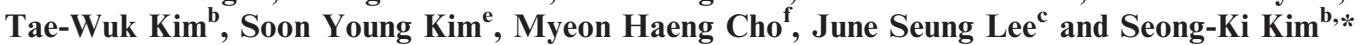 \\ ${ }^{\mathrm{a}}$ University College, ${ }^{\mathrm{f}}$ Department of Biology, Yonsei University, Seoul 120-749, Korea, \\ ${ }^{\mathrm{b}}$ Department of Life Science, Chung-Ang University, Seoul 156-756, Korea \\ 'Department of Biological Science, Ewha Woman's University, Seoul 120-750, Korea \\ ${ }^{\mathrm{d}}$ Department of Molecular, Cellular, and Developmental Biology, University of Michigan, Ann Arbor, MI 48109-1048, USA \\ e Department of Biological Science, Andong National University, Andong 760-749, Korea \\ ${ }^{I}$ These authors contributed equally to this work. \\ *Corresponding author, e-mail: skkimbio@cau.ac.kr
}

Received 16 December 2003; revised 2 March 2004

This study examines how brassinolide (BL) and ethylene interact in the gravitropic response mechanism of maize (Zea mays) primary roots. When applied exogenously, ethylene increases the rate of gravitropic curvature in a dose-dependent manner. This effect of ethylene was confirmed by the fact that AVG, a specific action inhibitor of ACC synthase, reduces the gravitropic curvature in the presence and absence of BL. Since AVG did not inhibit BL-increased gravitropic curvature completely, we investigated the possibility that BL may act on the gravitropic response by ways other than simply through enhanced ethylene production. We show that BL exhibits some of its stimulatory effect in the absence of ethylene. In addition, BL reduces the presentation time and lag period for the gravitropic response, whereas ethylene increases them. One possible mechanism of such action is that BL affects protein kinase activity, since the protein kinase inhibitors, staurosporine and H89, reduce BL-increased gravitropic curvature. In summary, $\mathrm{BL}$ is involved in the gravitropic response in maize primary roots via ethylene production, but it acts in a way that differs somewhat from that of ethylene.

\section{Introduction}

Gravitropism is a directional movement of plants in response to the gravity vector. The mechanism of the gravitropic response can be explained in terms of perception, signal transduction and asymmetric growth response, where gravity and several plant hormones are the signals (Kaufman et al. 1995). Auxins, cytokinins, and ethylene are hormones known to be involved in the gravitropic response. Of these, auxin is considered to be the primary hormone in this response (Kaufman et al. 1995). This is because gravitropism-induced differential cell elongation is mediated primarily by auxin, whose lateral redistribution is induced by gravistimulation treatment (Chen et al. 1999). Up to now, the role of auxin in the gravitropic response mechanism has been investigated most extensively in connection with its transport and gravity-signal transduction (Muday 2001, Ottenschlage et al. 2003).

Ethylene is also known to play an important role in the gravitropic response, in spite of the fact that there are opposing reports based on the relationship between the gravitropic response and ethylene production (Kaufman et al. 1985, Harrison and Pickard 1986, Balatti and Willemoes 1989, Woltering 1991). It has been reported that ethylene is responsible for decreased starch levels in the columella cells, and thus, possibly results in inhibition of the gravitropic response of Arabidopsis roots (Guisinger and Kiss 1999). In other studies, ethylene was reported to stimulate the gravitropic response in

Abbreviations - ACC, 1-aminocyclopropane-1-carboxylic acid; AVG, 1-alpha-(2-aminoethoxyvinyl)glycine; H89, $N$-(2-[p-Bromocinnamylamino]ethyl)-5-isoquinolinesulphonamide hydrochloride; IAA, indole-3-acetic acid; NPA, $N$-(naphth-1-yl)phthalamic acid; TIBA, 2,3,5triiodobenzoic acid 
tomato seedlings (Zobel 1973), dandelion plants (Clifford and Oxlade 1989), and snapdragon spikes (Philosoph-Hadas et al. 1996). Furthermore, it has been reported that this hormone redirects etiolated pea shoots to grow downward rather than upward upon gravistimulation (Burg and Kang 1993). Using the ethyleneinsensitive mutant, ein2-1, Golan et al. (1996) show that ethylene mediates the positive action of cytokinin in gravitropism of Arabidopsis seedlings. Recently, Madlung et al. (1999) showed that ethylene is necessary for a full gravitropic response of tomato seedlings, although it did not exert a primary role in the response. However, to our knowledge, the effects of ethylene on the gravitropic response of roots are not so well-established compared with that of shoots.

Following the isolation of brassinolide (BL) from rape pollen by Grove et al. (1979), more than 40 brassinosteroids (BRs) have been identified. Because they are found to occur ubiquitously in the plant kingdom, and are known to exert their roles at lower than micromolar concentrations, they are now considered to be a sixth class of plant hormones (Sasse 1990, Sakurai and Fujioka 1993, Yokota 1997). The roles of BRs in various events of plant growth and regulation have been well-characterized (Yokota 1997, Altman 1999, Li and Chory 1999). Many responses induced by BRs have been reported to be similar to those induced by auxin. Furthermore, some auxin-induced responses are synergistically enhanced by BRs. This indicates that both hormones are interactive in some aspects of plant growth and development (Yopp et al. 1981, Takeno and Pharis 1982, Cohen and Meudt 1983, Katsumi 1985, Eun et al. 1989, Kim et al. 1990, Fujii et al. 1991, Fujioka et al. 1998, Sasse 1999). BRs are also known to interact with ethylene. One of the well-known interactions is that BRs affect ethylene biosynthesis by increasing ACC synthase activity (Mandava 1988, Sakurai and Fujioka 1993).

It has been reported that the gravitropic curvature of bean (Meudt 1987) and tomato hypocotyls (Park 1998) is enhanced by the application of BRs, indicating that BRs may participate in the regulation of shoot gravitropism. These findings raise the possibility that BRs may also be involved in the regulation of root gravitropism. This led us to examine the effects of BRs on the gravitropic response in maize (Zea mays) primary roots, a system whose gravitropic response has been well-characterized. In our previous work (Kim et al. 2000), we showed that BL has a stimulatory effect on the gravitropic response, and that this effect was diminished and/or disappeared in the presence of auxin transport inhibitors such as NPA and TIBA. In addition, the stimulatory effect of BL is more pronounced in the presence of IAA. Thus, it is possible that the BL effect is auxin-dependent, and there may be a link between BL and IAA in the gravitropic response signal transduction pathway.

This current study was undertaken to test the hypothesis that BL may be involved in the root gravitropic response of maize via IAA-induced ethylene biosynthesis. In connection with this hypothesis, we attempt to explain the mode of BL action in relation to IAA and ethylene in the gravitropic signal transduction pathway in maize roots. Here, we show that BL stimulates the gravitropic response, in part, by eliciting an increase in ethylene level. We also conclude that BL exerts its role in a partially different way from that of ethylene in the gravitropic response mechanism.

\section{Materials and methods}

\section{Plant materials and chemicals}

Maize (Zea mays L. cv. Golden Cross Bantam) seeds were prepared and seedlings were grown as described in Kim et al. (2000). Seeds were washed several times with tap water and soaked in distilled water for $24 \mathrm{~h}$. After soaking, the seeds were placed in trays $(27 \times 20 \times 2.5 \mathrm{~cm})$ on water-saturated paper towels. To keep the seeds moist, they were covered with one more layer of watersaturated paper towels. The trays were then positioned vertically at $28 \pm 1{ }^{\circ} \mathrm{C}$ in the dark with $70 \% \mathrm{RH}$. After germination in the dark for 2 days, seedlings with $1.5-2 \mathrm{~cm}$ long straight primary roots were selected and used for the experiments.

For treatments of chemicals, stock solutions were prepared as follows. BL, staurosporine, and H89 were dissolved in DMSO, while AVG was dissolved in distilled water. For every treatment and for the control, the final concentration of DMSO was $0.1 \%(\mathrm{v} / \mathrm{v})$. At this concentration, no adverse side-effects were found in this study. All chemicals used in this study were obtained from Sigma Chemical Co. (St. Louis, MO, USA).

\section{Measurement of gravitropic curvature of maize primary roots}

The root caps of seedlings were immersed in the buffer solution (5 mM MES-Tris, pH 6.8) containing various concentrations of $\mathrm{BRs}$ for $2 \mathrm{~h}$ with aeration at $25 \pm 1{ }^{\circ} \mathrm{C}$. The hormone-treated roots were then placed horizontally in a lucent Plexiglas ${ }^{\mathrm{TM}}$ container $(13 \times 9 \times$ $6 \mathrm{~cm}$ ) for gravistimulation. Using a syringe, ethylene was injected into a chamber in which the container was located.

For early kinetics experiments, a closed circuit digital camera and a computer program, Image-Pro Plus, made by Yongma (Seoul, Korea) were used. The image of the root was magnified 10 times on a monitor and recorded every $1 \mathrm{~min}$ for up to $1 \mathrm{~h}$. The angles of curvature were measured after the gravistimulation treatment, using this program.

For determination of the presentation time, maize roots were gravistimulated for $0,5,10,15$ and $20 \mathrm{~min}$ after the pretreatment of hormones as above and then rotated on a clinostat (1 r.p.m) for $30 \mathrm{~min}$. A plot of curvature vs. the time for gravistimulation was made. The intercept of the line on the $x$ axis was taken to be an estimation of the presentation time. 


\section{Measurement of ethylene content}

One $\mathrm{ml}$ of air was withdrawn with a syringe from a vial containing $1 \mathrm{ml}$ of potassium phosphate buffer $(50 \mathrm{mM}$, $\mathrm{pH}$ 6.8) plus the experimental roots and injected into a gas chromatograph equipped with a column containing Porapac Q (Shimadzu GC-8A, Japan). Vials containing the roots plus buffer were incubated at $27 \pm 1^{\circ} \mathrm{C}$ in the dark.

\section{Activity assays of ACC synthase and ACC oxidase}

Activity of ACC synthase was assayed as described by Woeste et al. 1999). Root segments (4 g) were ground on ice with $8 \mathrm{ml}$ of $250 \mathrm{mM}$ potassium phosphate buffer ( $\mathrm{pH}$ 8.0) containing $10 \mu M$ pyridoxal phosphate, $1 \mathrm{mM}$ EDTA, $2 \mathrm{~m} M$ PMSF, and $5 \mathrm{~m} M$ DTT. The homogenate was kept for $4 \mathrm{~min}$ on ice and centrifuged for $15 \mathrm{~min}$ at $15000 \mathrm{~g}$. One $\mathrm{ml}$ of the supernatant was incubated with $5 \mathrm{~m} M$ S-AdoMet $(0.1 \mathrm{ml})$ for $1 \mathrm{~h}$ at $22^{\circ} \mathrm{C} \pm 1^{\circ} \mathrm{C}$ and placed on ice with $0.1 \mathrm{ml}$ of $20 \mathrm{mM} \mathrm{HgCl} 2$ and $0.1 \mathrm{ml}$ of $\mathrm{NaOH} / \mathrm{NaOCl}(1: 1)$ mixture for $10 \mathrm{~min}$. The ethylene production from this mixture was used for determination of ACC synthase activity (Lizada and Yang 1979).

The ACC oxidase activity assay was carried out in vivo according to the method of Wang and Woodson (1989). Forty root segments were incubated in a $50 \mathrm{~m} M$ potassium buffer (pH 6.8) containing $0.1 \mathrm{~m} M$ aminoethoxyvinylglycine (AVG) in the presence or absence of brassinolide (BL). After the incubation, root segments were washed with distilled water, then infiltrated with $1 \mathrm{~m} M$ ACC for $2 \mathrm{~h}$ at $27 \pm 1^{\circ} \mathrm{C}$ in the dark. The ACC oxidase activity determination was based on ethylene produced from primary root segments placed in distilled water for $1 \mathrm{~h}$ in the absence of ACC.

\section{Replication of experiments and statistical analysis of data}

All experiments for gravitropic curvature and elongation of maize primary roots were performed at least three times. In every experiment, 20 primary roots were used. A $P$-value of $<0.05$ was considered to be significant. To test for significance of the data, mean values were calculated with Student's $t$-test.

\section{Results}

\section{BL increases ethylene production and the gravitropic response}

Our previous work shows that BL interacts with IAA in the activation of the root gravitropic response (Kim et al. 2000). This implies that BL acts at one or more auxin action site(s). Among these, one possibility is that the BL participates in the response by affecting IAA-induced ethylene production. This was tested by examining BL effects on activities of ethylene biosynthetic enzymes, including ACC synthase and ACC oxidase. Results show that BL not only increases ethylene production synergistically with IAA (Lim et al. 2002), but also stimulates the activities of ACC synthase and ACC oxidase in a dose-dependent manner (Fig. 1A). At $0.1 \mu M$, BL increased the activity of ACC oxidase $(55 \%)$ more than that of ACC synthase $(23 \%)$.

Is there any relationship between BL-induced ethylene biosynthesis and the gravitropic response? To answer this question, a dose-response analysis of the effect of $\mathrm{BL}$ on ethylene synthesis and the gravitropic response was performed. The gravitropic curvature and ethylene content were increased by BL in a dose-dependent manner, although the patterns of these increases were not perfectly matched with each other (Fig. 1B). For example, at $10^{-7} M$, BL increased the gravitropic curvature by $33 \%$, while it enhanced ethylene production by $11 \%$.

These results suggest that ethylene exerts a stimulatory role in the gravitropic response in primary roots of maize. In order to test this possibility, we measured the curvature of roots gravistimulated in the presence of various concentrations of ethylene. Fig. 2 shows that ethylene increases the gravitropic curvature in a concentration-dependent manner. Ethylene at $0.1 \mu \mathrm{l} \cdot 1^{-1}$ increases the curvature by $59 \%$. It did not show any

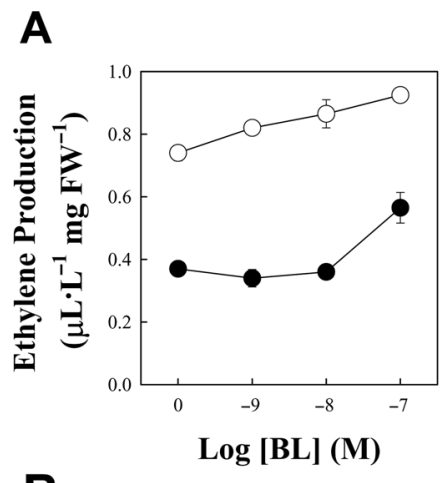

B

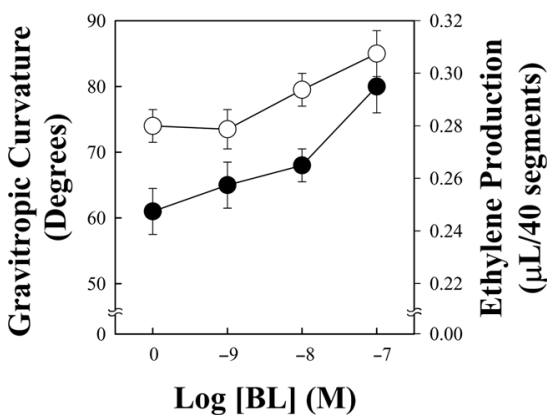

Fig. 1. Dose-responses for BL effects on ethylene production and the gravitropic response. In (A), activities of ACC synthase (•) and ACC oxidase (O) in maize roots are compared. Activity of ACC synthase was determined by estimation of ACC content by the use of crude extracts of roots incubated for $8 \mathrm{~h}$ in the presence of various concentrations of BL. ACC oxidase activity was assayed using intact root segments incubated in a solution containing 0.1 $\mathrm{m} M \mathrm{AVG}$ and $\mathrm{BL}$ for $8 \mathrm{~h}$. In (B), dose-responses for BL effects on the gravitropic response $(\mathrm{O})$ and ethylene production $(\bullet)$ were examined. Maize roots were placed horizontally for $4 \mathrm{~h}$ at room temperature after preincubation in $5 \mathrm{~m} M$ MES-Tris ( $\mathrm{pH}$ 6.8) buffer containing various concentrations of BL for $2 \mathrm{~h}$. Symbols denote mean values \pm SE of five (A) or four (B) independent experiments. 
A
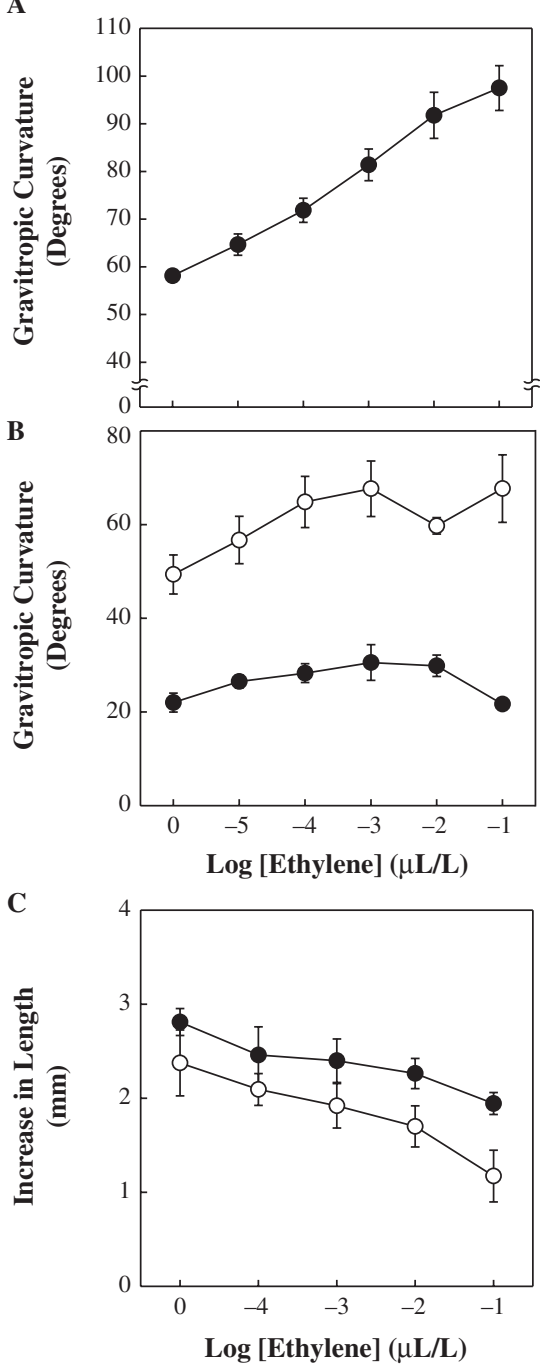

Fig. 2. Dose-response for ethylene effects on the gravitropic response (A and $\mathrm{B}$ ) and elongation $(\mathrm{C})$ in maize roots. The roots were gravistimulated by placing them horizontally in the presence or absence of various concentrations of ethylene for $3 \mathrm{~h}$ in the light at room temperature (A). In (B), the same experiments were performed in the presence of AVG $(10 \mu M) \pm \mathrm{BL}(0.1 \mu M)$. In $(\mathrm{C})$, root lengths were measured after the roots were incubated in various concentrations of ethylene in the presence or absence of AVG $(10 \mu M)$ for $3 \mathrm{~h}$. Bars in the graph denote SE of four independent experiments using 120 segments. Open and closed circles in (B) and (C) denote treated with AVG + BL and with AVG only, respectively.

further enhancing effect at concentrations greater than $0.1 \mu 1 \cdot \cdot^{-1}$ (data not shown), indicating that the effect was saturated above this concentration. However, the hormone decreased length of the roots in a dose-dependent manner (Fig. 2C). In order to confirm the ethylene effect, we performed the same experiments as above, but under conditions where endogenous ethylene was removed with AVG, a specific inhibitor of ACC synthase. An optimal dose-response curve of the ethylene effect is shown in Fig. 2B. It peaks at $0.001 \mu l \cdot 1^{-1}$ (lower line of Fig. 2B). However, ethylene decreased root elongation, once again, but to a lesser extent in the presence of AVG than in its absence. These results confirm the view that ethylene stimulates the gravitropic response, although the stimulatory effect is small (c. 35\%) when compared with the effects shown in the absence of the inhibitor, AVG.

\section{BL-increases the gravitropic response in the presence or absence of AVG}

The possibility that BL exerts its effect via ethylene production in the gravitropic response was probed by the use of AVG. This inhibitor exhibited a maximum inhibitory effect on ethylene production at $0.03 \mathrm{mM}$ and above. Upon treatment with $0.1 \mathrm{~m} M$ AVG, BL-increased gravitropic curvature was reduced (Fig. 3A). The gravitropic curvature of the control was also reduced. AVG reduced the gravitropic curvature of the control and BL-increased curvature by 48 and $20 \%$, respectively (compare 1 st and 3 rd columns for the control and compare 2nd and 4th columns for BL-treated). These results support the notion that BL-increased gravitropic response is mediated by BL-increased ethylene levels.
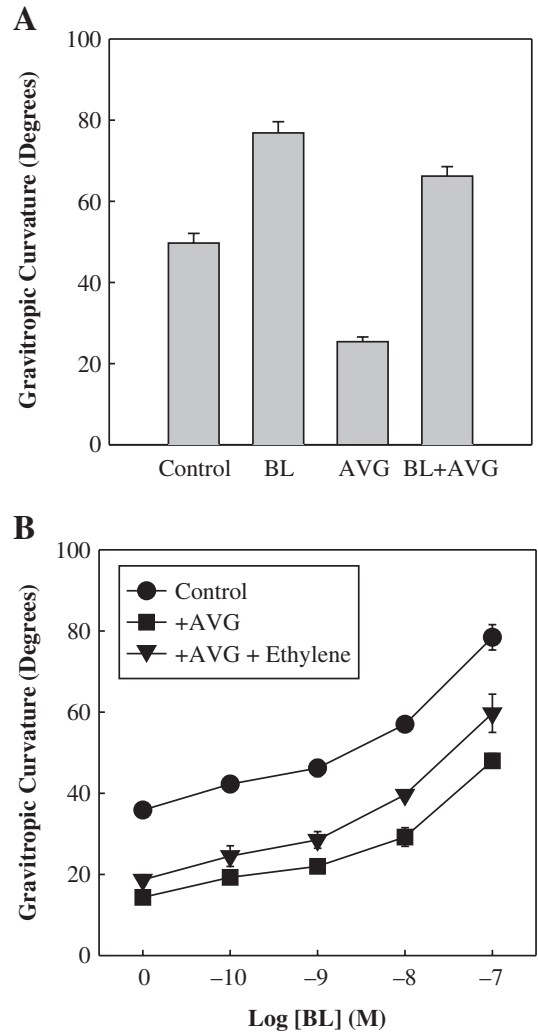

Fig. 3. Effect of AVG on the gravitropic response of maize roots in the presence or absence of BL. Maize roots were preincubated in $5 \mathrm{~m} M$ MES-Tris ( $\mathrm{pH}$ 6.8) buffer in the presence or absence of BL $(0.1$ $\mu M)$ and/or AVG $(10 \mu M)$ for $2 \mathrm{~h}$ at room temperature (A). In (B), the roots were preincubated in solutions containing $5 \mathrm{~m} M$ MES-Tris (pH 6.8) buffer, BL $\left(0-10^{-7} M\right) \pm$ ethylene $\left(0.1 \mu \mathrm{l}^{-1}\right)$ and/or AVG $(10 \mu M)$ for $2 \mathrm{~h}$ at room temperature. After the preincubation, the roots were placed horizontally for $3 \mathrm{~h}$. Bars in the graph denote SE of four independent experiments using 120 segments. 
However, the curvature of the roots treated with AVG and $\mathrm{BL}$ was greater than that of the control. This result means that $\mathrm{BL}$ can still stimulate the gravitropic response in the presence or absence of a low level of activity of ACC synthase, and that BL-increased gravitropic curvature is not fully inhibited by AVG. However, it is evident that BL stimulated the gravitropic response, in part, via an increase in ethylene level. This observation suggests that BL may affect action site(s) other than those associated with auxin-induced ethylene production in the gravitropic response. Therefore, this speculation was tested by examining the dose-response of the BL effect on the gravitropic response in the presence or absence of AVG and/or ethylene. In all cases, the amount of curvature was increased with increasing BL concentrations (Fig. 3B). By increasing its concentration, BL enhanced the gravitropic curvature, even in the presence of AVG. This indicates that BL may affect the response under conditions where the level of endogenous ethylene is low or absent (see $\mathbf{i n}$ Fig. 3B). This result also shows that AVG decreased the BL effect, whereas ethylene increased the effect. This implies that ethylene may influence the responsiveness of the roots to BL upon gravistimulation. In addition, the stimulatory effect of BL on the gravitropic response is evident at any concentration of ethylene applied (upper line of Fig. 2B). Taken together, these results indicate that BL seems to affect the gravitropic response via ethylene, as well as independently.

\section{BL increases the sensitivity of roots to gravity and is involved early in the gravitropic response process}

If $\mathrm{BL}$ can exert its role independently, the action modes of $\mathrm{BL}$ and ethylene may be different. In order to test this assumption, and therefore obtain more information on the BL effect, we determined if BL affects the sensitivity of the roots to gravity. As shown in Fig. 4A, the presentation time of BL-treated roots was about $1.2 \mathrm{~min}$, while that of the control roots was about $3.4 \mathrm{~min}$. This result indicates that the amount of time needed for the gravity signal to initiate a gravitropic response of the roots is reduced by BL. This implies that the sensitivity of the roots to gravity is increased by BL. However, ethylene caused a delay (ca. $4.6 \mathrm{~min}$ ) in the presentation time. The early kinetics for the BL effect on the gravitropic response was also studied. The lag period for the gravitropic response was about $6.5 \mathrm{~min}$ for $\mathrm{BL}$ treatment, $20.8 \mathrm{~min}$ for ethylene treatment, and about $8.5 \mathrm{~min}$ for the control (Fig. 4B and Table 1). However, it should be noted that this inhibitory effect of ethylene was reversed around $60 \mathrm{~min}$ after the gravistimulation treatment, and thus, the hormone resulted in an increase in the gravitropic curvature response after $60 \mathrm{~min}$ of treatment, as shown in Figs 1, 2, and 3 (see inset in Fig. 4B). In addition, the initial rate (during 25-40 min after the start of gravistimulation) of gravitropic curvature was $0.46^{\circ} \cdot \mathrm{min}^{-1}$ in the presence of $\mathrm{BL}$ while it was $0.29^{\circ} \cdot \min ^{-1}$ in the absence of the hormone (Table 1 ). But again, ethylene reduced the initial rate of the gravi-
$\mathbf{A}$

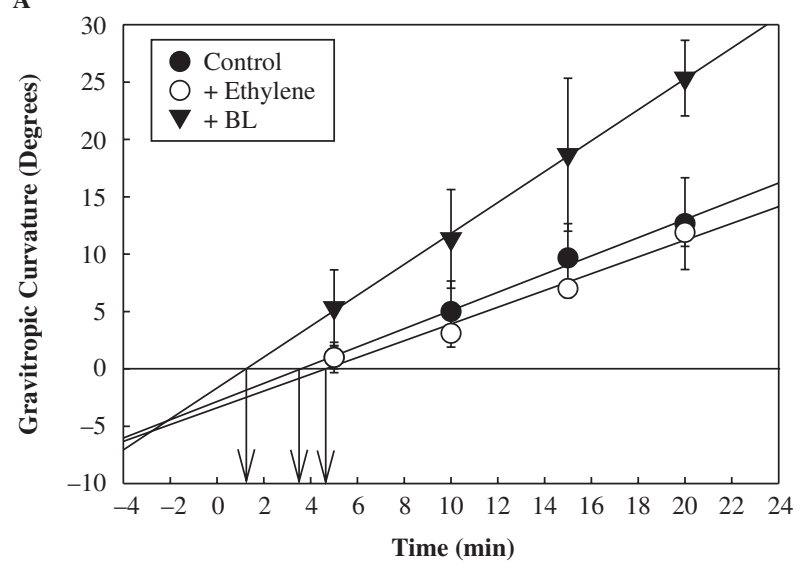

B

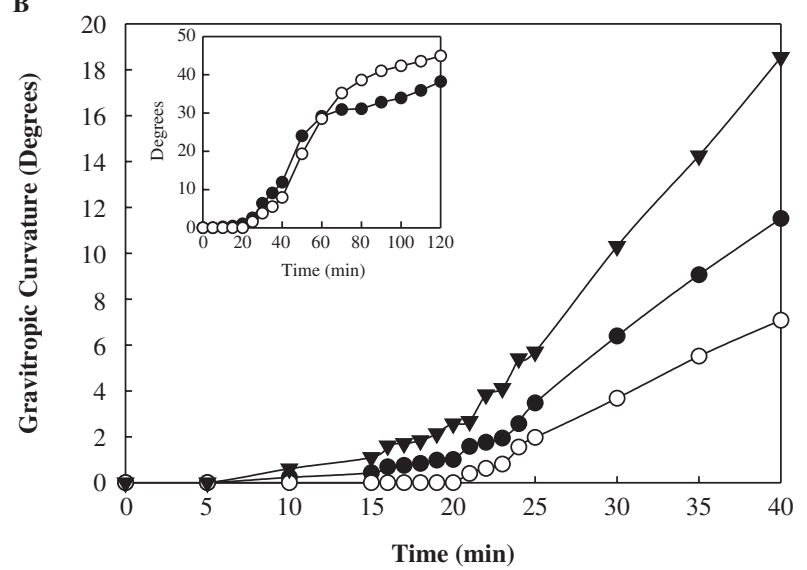

Fig. 4. Effect of BL on the presentation time (A) and early kinetics for BL effect on the gravitropic response (B). For determination of the presentation time (A), maize roots were preincubated as above and gravistimulated for 5, 10, 15, and $20 \mathrm{~min}$. After the gravistimulation, the roots were subjected to clinorotation treatment for $30 \mathrm{~min}$. BL-treated roots are statistically different from the ethylene-treated roots as determined by Student's $t$-test at $>0.1$. For early kinetic studies (B), the changes of the gravitropic curvatures of 18 segments were chased using computer-aided CCD cameras (see Materials and methods). Maize roots were preincubated in $5 \mathrm{mM}$ Mes-Tris buffer ( $\mathrm{pH}$ 6.8) with or without $10^{-7} \mathrm{M} \mathrm{BL}$ or $0.1 \mu \mathrm{l} \cdot \mathrm{1}^{-1}$ ethylene for $2 \mathrm{~h}$ and placed horizontally for $40 \mathrm{~min}$ at room temperature. The inset shows the long-term kinetics of ethylene effect. The bars in the graphs denote SE of four independent experiments using 18-20 segments per treatment.

tropic curvature down to $0.18^{\circ} \cdot \mathrm{min}^{-1}$. Taken together, these results indicate that $\mathrm{BL}$ tends to increase the sensitivity of the roots to the gravity vector and the early gravitropic responsiveness of the roots, while ethylene does the opposite.

\section{Protein kinase inhibitors reduce the BL- and ethylene- increased gravitropic response in different ways}

The BL-increased sensitivity of roots to gravity suggests that $\mathrm{BL}$ is involved in the gravity signal transduction pathway. To explore this possibility, we studied the effects of protein kinase inhibitors on the BL-induced gravitropic response. Staurosporine, an inhibitor of 
Table 1. Effect of BL and ethylene on early kinetics for the gravitropic response. The changes of the gravitropic curvatures of roots were monitored by using computer-aided CCD cameras (see Materials and methods). Maize roots were preincubated in $5 \mathrm{mM}$ Mes-Tris buffer ( $\mathrm{pH}$ 6.8) with or without $10^{-7} \mathrm{M}$ BL or $0.1 \mathrm{ppm}$ ethylene and placed horizontally for $40 \mathrm{~min}$ at room temperature. The lag period and rate of initial growth denote the duration from the initiation of the gravistimulation to the first gravitropic curvature and the increase in the rate of curvature during 25-40-min period after the start of the gravistimulation, respectively. The SE was calculated from data of four independent experiments using 18-20 roots per treatment.

\begin{tabular}{lcc}
\hline Lag period & $\begin{array}{l}\text { Rate of initial } \\
\text { growth }(\mathrm{min})\end{array}$ & $\left(\right.$ Degrees $\left.\mathrm{min}^{-1}\right)$ \\
\hline Control & $8.50 \pm 1.30$ & $0.298 \pm 0.026$ \\
+ Ethylene & $20.81 \pm 0.21$ & $0.176 \pm 0.033$ \\
+ Brassinolide & $6.50 \pm 0.76$ & $0.463 \pm 0.022$ \\
\hline
\end{tabular}

protein kinase activity, decreases the gravitropic curvature of the control plants by about $75 \%$ (Fig. 5). Such an inhibitory effect was also evident in the presence of ethylene. The amount of inhibition (ca. 90\%) was more than that for BL-treated roots (ca. 75\%). In addition, in the presence of staurosporine, ethylene-induced gravitropic curvature was smaller than that of the control, whereas BL-treated roots showed greater curvature than that of the control. A similar pattern of inhibition was observed when $\mathrm{H} 89$, another protein kinase inhibitor, was applied (data not shown). Taken together, these results indicate that $\mathrm{BL}$ and ethylene exhibit different patterns of response to protein kinase inhibitors. This implies that the two hormones exert their roles in different ways in the gravity signal-transduction pathway.

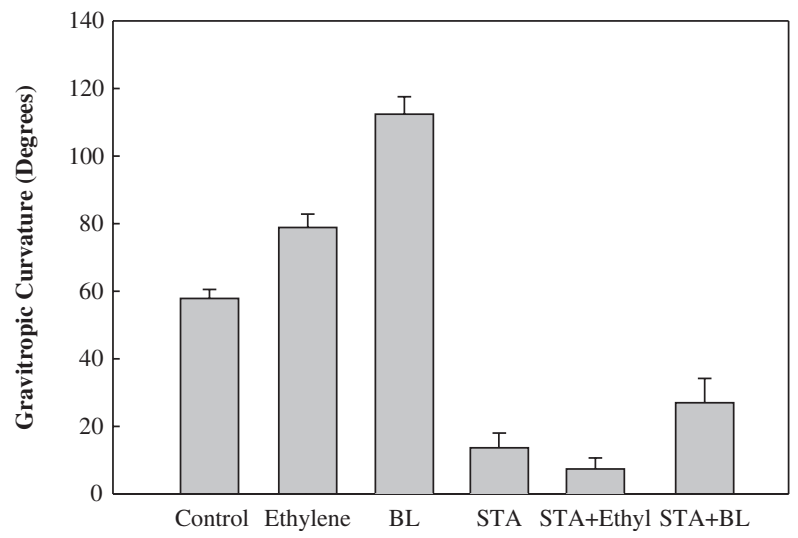

Fig. 5. Effect of staurosporine (STA) on the gravitropic response in the presence or absence of BL or ethylene. Maize primary roots were preincubated in solutions containing $5 \mathrm{mM}$ Mes-Tris $(\mathrm{pH} 6.8) \pm 10^{-6} M$ STA, in the presence or absence of $10^{-7} M \mathrm{BL}$, or the roots were preincubated in the same solution except that 0.1 $\mu \mathrm{l} \cdot \mathrm{l}^{-1}$ ethylene was added or not added instead of BL for $2 \mathrm{~h}$. After the preincubation, the roots were placed horizontally for $3 \mathrm{~h}$ for gravistimulation treatment. Values in the graphs denote the means $\pm \mathrm{SE}$ of five independent experiments

\section{Discussion}

There are many reports on hormone interactions in connection with many physiological processes in plants (Akiyoshi et al. 1983, Abeles et al. 1992, Liang et al. 1992, Yang et al. 1996, Bethke et al. 1997, Vogel et al. 1998, Ross and O'Neill 2001). In relation to the gravitropic response, we showed that $\mathrm{BL}$ increases the response and that the stimulatory effect is greatly diminished by auxin transport inhibitors such as NPA and TIBA (Kim et al. 2000). We also showed that BL interacts with IAA by increasing the sensitivity of the roots to the auxin in this response. Based on these facts, we have attempted to unravel the relationship between $\mathrm{BL}$ and IAA in the gravitropic response mechanism more completely. First, we examined the possibility that BL acts via auxin-induced ethylene biosynthesis. In this case, BL increased the activity of ACC synthase (Fig. 1A). This implies that BL affects ethylene biosynthesis through an interaction with IAA. This assumption is supported by the fact that AVG, a specific inhibitor of ACC synthase, reduces BL-increased gravitropic curvature (Fig. 3). In addition, Lim et al. (2002) reported that BL and IAA increased ethylene production in a synergistic manner. Results depicted in Fig. 1A also show that BL increases the activity of ACC oxidase, another ethylene biosynthesis enzyme. However, when the roots were treated with $\mathrm{Co}^{2+}$, an ACC oxidase inhibitor, BL-induced gravitropic curvature was not reduced significantly (data not shown) indicating that BL-increased activity of ACC oxidase may not be a significant factor in the gravitropic response. In conclusion, we deduced that one of the $\mathrm{BL}$ effects in the gravitropic response mechanism is manifested through auxin-induced ethylene biosynthesis.

Second, we also determined if BL exerts its role in the gravitropic response in ways other than through ethylene production. Several lines of evidence show that BL exerts its effect in gravitropic curvature in the absence or with very small amounts of ethylene. BL still increased the gravitropic response in the presence of exogenously applied AVG (Fig. 3A) and ethylene (Figs 2B and 3B). Further, the action mode of the two hormones in the gravitropic response is different based on the fact that their respective presentation times and early growth kinetic parameters for gravitropic curvature initiation are not the same (Fig. 4). The effect of BL also differs from that of ethylene on the gravitropic response in the presence of protein kinase inhibitors such as staurosporine and H89 (Fig. 5). These two results imply that BL and ethylene differ in the ways that they modulate the gravity perception and signal transduction processes in maize roots. Taken together, it is concluded that $\mathrm{BL}$ interacts with auxin in the gravitropic response by enhancing ethylene biosynthesis, and that its mode of action is not entirely different from that of ethylene.

In the present study, we show that ethylene increases the gravitropic curvature in a dose-dependent manner (Fig. 2A) and that AVG decreases the curvature significantly (Fig. 3). These results confirm the observation that 
ethylene plays a positive effector role in the gravitropic response of maize roots, as also established for tomato seedlings (Zobel 1973) and hypocotyls (Madlung et al. 1999), dandelion scapes (Clifford and Oxlade 1989), and snapdragon spikes (Philosoph-Hadas et al. 1996). Results in Fig. 3B also suggest that ethylene may affect the responsiveness of roots to BL. If this is true, ethylene can act as a modulator of BL-increased gravitropic response. Furthermore, ethylene causes an increase in the presentation time (Fig. 4A) and shows no stimulatory effect on the gravitropic response in the presence of the protein kinase action inhibitor, staurosporine (Fig. 5). These facts strongly suggest that ethylene is involved in gravity perception and the gravity signal transduction pathway. Taken together, ethylene seems to be an essential player in the gravitropic response. However, we are not convinced that ethylene exerts a primary role in the response. More evidence is needed to support the hypothesis that ethylene is required for achievement of a full gravitropic curvature response in maize roots, as discussed by Madlung et al. (1999).

In this study, we compared the presentation time in order to compare responsiveness (or sensitivity) of roots to gravistimulation treatment based upon suggested significance of the presentation time (Casper and Pickard 1989, Kiss et al. 1989, Sack 1991). Recently, Perbal et al. (2002) points out that the logarithmic (L) model that we employed in this study, generally does not fit the experimental data that have been published. Instead, they suggest that a hyperbolic $(\mathrm{H})$ model is a more acceptable model based on comparing correlation coefficients (r) in roots of many plants including Arabidopsis, Lens culinaris, and Lepidium sativum. Our statistical calculation estimates that $r$ of the control in Fig. 4B is 0.962 and 0.986 for the $\mathrm{H}$ and $\mathrm{L}$ models, respectively. This means that the $\mathrm{L}$ model is more applicable to our experimental data than the $\mathrm{H}$ model is. However, it should be mentioned that the $\mathrm{H}$ model is valuable in explaining the gravisensitivity with other parameters (Perbal et al. 2002).

In the present study, we found that AVG has a significant inhibitory effect on the gravitropic response in maize roots and that this inhibitory effect does not fully recover, when high concentrations of ethylene $\left(0.1 \mu 1 \cdot 1^{-1}\right)$ are applied. This could be due to a significant decrease in the number of endogenous ethylene molecules that are available for action, such as in ethylene receptor binding. However, more work is needed to test this assumption.

Based on results from the present and previous studies, we propose the following working model. BL increases the gravitropic response in roots only in the presence of auxin. It also increases tissue sensitivity to auxin (Kim et al. 2000). BL acts by stimulating auxin-induced ACC synthase activity with consequent enhancement in ethylene biosynthesis. The higher levels of ethylene lead to an increase in gravitropic curvature. At the same time, ethylene enhances the responsiveness of roots to BL during the gravitropic response. BL plays a regulatory role in gravity perception and signal transduction, but it does so in ways that are independent of ethylene.

This model can now be rigorously tested with roots of Arabidopsis thaliana because of the availability of BL perception mutants, such as bril (Li and Chory 1997) and bakl (Nam and Li 2002). In future work, we will thus focus on the gravitropic response of Arabidopsis roots in order to unravel BL's effects on the gravitropic response mechanism in relation to the other participating hormones, namely, ethylene and auxin.

Acknowledgements - This work is supported by Grant no. R01-2002-000-00367-0 (to S-KK, Y-SK, HSY) from KOSEF, by IS-3133-99C from BARD, the United States-Israel Binational Agricultural Research and Development Fund (to PBK, SCC), and by CG-134 from CFGC, Crop Functional Genomics Center of the 21st Century Frontier Research Program funded by the Ministry of Science and Technology (MOST) and Rural Development Administration (RDA) of Republic of Korea (to MHC).

\section{References}

Abeles FB, Morgan PW, Saltveit ME JR (1992) Ethylene in Plant Biology, 2nd edn. Academic Press, San Diego.

Akiyoshi DE, Morris RO, Hinz R, Mischke BS, Kosuge T, Garkinkel DJ, Gordon MP, Nester EW (1983) Cytokinin/ auxin balance in grown gall tumors is regulated by specific loci in the T-DNA. Proc Natl Acad Sci USA 80: 407-411

Altman T (1999) Molecular physiology of brassinosteroids revealed by the analysis of mutants. Planta 208: 1-11

Balatti PA, Willemoes JG (1989) Role of ethylene in the geotropic response of bermuda grass (Cynodon dactylon L. Pers.) stolons. Plant Physiol 91: 1251-1254

Bethke PC, Schuurink R, Jones RL (1997) Hormonal signaling in cereal aleurone. J Exp Bot 48: 1337-1356

Burg SP, Kang BG (1993) Gravity dependent ethylene action. In: Pech JC, Latache A, Balague C, eds. Cellular and Molecular Aspects of the Plant Hormone Ethylene. Kluwer Academic Publishers, Dordrecht, The Netherlands, pp 335-341

Casper T, Pickard BG (1989) Gravitropism in a starchless mutant of Arabidopsis. Implications for the starch statolith theory of gravity sensing. Planta 177: 185-197

Chen R, Rosen E, Masson PH (1999) Gravitropism in higher plants. Plant Physiol 120: 343-350

Clifford PE, Oxlade EL (1989) Ethylene production, georesponse, and extension growth in dandelion peduncles. Can J Bot 67: 1927-1929

Cohen JD, Meudt WT (1983) Investigations on the mechanism of the brassinosteroid response. I. Indole-3-acetic acid metabolism and transport. Plant Physiol 72: 691-694

Eun J-S, Kuraishi S, Sakurai N (1989) Changes in levels of auxin and abscisic acid and the evolution of ethylene in squash hypocotyls after treatment with brassinolide. Plant Cell Physiol 30: $807-810$

Fujii S, Hirai K, Saka H (1991) Growth-regulating action of brassinolide in rice plant. In: Cutler HG, Yokota T, Adam G, eds. Brassinosteroids, Chemistry, Bioactivity, and Application, ACS Symposium Series 474. American Chemical Society, Washington DC, pp 306-311

Fujioka S, Noguchi T, Takatsuto S, Yoshida S (1998) Activity of brassinosteroids in the dwarf rice lamina inclination bioassay. Phytochem 49: 1841-1848

Golan A, Tepper M, Soudry E, Horwitz BA, Gepstein S (1996) Cytokinin, acting through ethylene, restores gravitropism to Arabidopsis seedlings grown under red light. Plant Physiol 112: 901-904

Grove MD, Spencer FG, Rohwededer WK, Mandava NB, Worley JF, Warthen JD Jr, Steffens GL, Flippen-Anderson JL, Cook JC Jr (1979) Brassinolide, a plant growth promoting steroid isolated from Brassica napus pollen. Nature 281: 216-217 
Guisinger MM, Kiss JZ (1999) The influence of microgravity and spaceflight on columellar cell ultrastructure in starch-deficient mutants of Arabidopsis. Am J Bot 86: 1357-1366

Harrison MA, Pickard BG (1986) Evaluation of ethylene as a mediator of gravitropism by tomato hypocotyls. Plant Physiol 80: $592-595$

Kastumi M (1985) Interaction of a brassinosteroid with IAA and $\mathrm{GA}_{3}$ in the elongation of cucumber hypocotyl sections. Plant Cell Physiol 26: 615-625

Kaufman PB, Pharis RP, Reid DM, Beall FD (1985) Investigation into the possible regulation of negative gravitropic curvature in intact Avena sativa plants and in isolated stem segments by ethylene and gibberellins. Physiol Plant 65: 237-244

Kaufman PB, Wu L-L, Brock TG, Kim D (1995) Hormones and the orientation of growth. In: Davis PJ, ed. Plant Hormones: Physiology, Biochemistry and Molecular Biology, 2nd edn. Kluwer Academic Publishers, Dordrecht, The Netherlands, pp 547-571

Kim S-K, Abe H, Little CHA, Pharis RP (1990) Identification of two brassinosteroids from cambial region of Scots pine (Pinus silverstris) by gas chromatography-mass spectrometry, after detection using a dwarf rice lamina inclination bioassay. Plant Physiol 94: 1709-1713

Kim S-K, Chang SC, Lee EJ, Chung W-S, Kim Y-S, Hwang S, Lee JS (2000) Involvement of brassinosteroids in the gravitropic response of primary root of maize. Plant Physiol 123: 997-1004

Kiss JZ, Hertel R, Sack FD (1989) Amyloplasts are necessary for full gravitropic sensitivity in roots of Arabidopsis thaliana. Planta 177: 198-206

Li J, Chory J (1997) A putative leucine-rich repeat receptor kinase involved in brassinosteroid signal transduction. Cell 90: 929-938

Li J, Chory J (1999) Brassinosteroid actions in plants. J Exp Bot 50: $332-340$

Liang X, Abel S, Keller J, Shen N, Theologis A (1992) The 1-aminocyclopropane-1-carboxylate synthase gene family of Arabidopsis thaliana. Proc Natl Acad Sci USA 89: 11046-11050

Lim SH, Chang SC, Lee JS, Kim S-K, Kim SY (2002) Brassinosteroids affect ethylene production in the primary roots of maize (Zea mays L.). J Plant Biol 45: 148-153

Lizada C, Yang SF (1979) A simple and sensitive assay for 1-aminocyclopropane-1-carboxylic acid. Ann Biochem 100: 140-145

Madlung A, Behringer FJ, Lomax TL (1999) Ethylene plays multiple non primary roles in modulating the gravitropic response in tomato. Plant Physiol 120: 897-906

Mandava BN (1988) Plant growth promoting brassinosteroids. Annu Rev Plant Physiol Plant Mol Biol 39: 23-52

Meudt WT (1987) Investigations on mechanism of brassinosteroid response. VI. Effect of brassinolide on gravitropism of bean hypocotyls. Plant Physiol 83: 195-198

Muday GK (2001) Auxins and tropisms. J Plant Growth Regul 20 226-243

Nam KH, Li J (2002) BRI1/BAK1, a receptor kinase pair mediating brassinosteroid signaling. Cell 110: 203-212
Ottenschlager I, Wolff P, Wolverton C, Bhalerao RP, Sandberg G, Ishikawa H, Evans M, Palme K (2003) Gravity-regulated differential auxin transport from columella to lateral root cap cells. Proc Natl Acad Sci U S A 100: 2987-2991

Park WJ (1998) Effect of epibrassinolide on hypocotyl growth of the tomato mutant diageotropica. Planta 207: 120-124

Perbal G, Jeune B, Lefranc A, Carnero-Diaz E, Driss-Ecole D (2002) The dose-response curve of the gravitropic reaction: a re-analysis. Physiol Plant 114: 336-342

Philosoph-Hadas S, Meir S, Rosenberger I, Halevy AH (1996) Regulation of the gravitropic response and ethylene biosynthesis in gravistimulated snapdragon spikes by calcium chelators and ethylene inhibitors. Plant Physiol 110: 301-310

Ross J, O'Neill D (2001) New interactions between classical plant hormones. Trends Plant Sci 6: 2-4

Sack FD (1991) Plant gravity sensing. Int Rev Cytol 127: 193-252

Sakurai A, Fujioka S (1993) The current status of physiology and biochemistry of brassinosteroids. Plant Growth Regul 13: $147-159$

Sasse JM (1990) Brassinolide-induced elongation and auxin. Plant Physiol 80: 401-408

Sasse JM (1999) Physiological actions of brassinosteroids. In: Sakurai A, Yokota T, Clouse SD, eds. Brassinosteroids: Steroidal Plant Hormone. Springer-Verlag, Tokyo, pp 306-311

Takeno K, Pharis RP (1982) Brassinosteroid-induced bending of the leaf lamina of dwarf rice seedlings: an auxin-mediated phenomenon. Plant Cell Physiol 23: 1275-1281

Vogel JP, Woeste K, Theologis A, Kieber JJ (1998) Recessive and dominant in the ACC synthase 5 gene of Arabidopsis results in cytokinin-insensitivity and ethylene overproduction respectively. Proc Natl Acad Sci USA 95: 4766-4771

Wang H, Woodson WR (1989) Reversible inhibition of ethylene action and interruption of petal senescence on carnation flowers by norbonadiene. Plant Physiol 89: 434-438

Woeste KE, Ye C, Kieber JJ (1999) Two Arabidopsis mutants that overproduce ethylene are affected in the posttranscriptional regulation of 1-aminocyclopropane-1-carboxylic acid synthase. Plant Physiol 119: 521-529

Woltering EJ (1991) Regulation of ethylene biosynthesis in gravistimulated Kniphofia (hybrid) flower stalks. J Plant Physiol 138: 443-444

Yang T, Davis PJ, Reid JB (1996) Genetic dissection of the relative roles of auxin and gibberellin in the regulation of stem elongation in intact light-grown peas. Plant Physiol 110: $1029-1034$

Yokota T (1997) The structure, biosynthesis and function of brassinosteroids. Trends Plant Sci 2: 137-143

Yopp JH, Mandava NB, Sasse JM (1981) Brassinolide a growth promoting steroidal lactone. I. Activity in selected auxin bioassays. Physiol Plant 53: 445-452

Zobel RW (1973) Some physiological characteristics of the ethylene-requiring tomato mutant diageotropica. Plant Physiol 52: 385-389 